\title{
GHz Serial Passive Clock Distribution in VLSI Using Bidirectional Signaling
}

\author{
V. Prodanov and M. Banu \\ MHI Consulting, LLC, New Jersey, USA
}

\begin{abstract}
We introduce a serial passive clock distribution technique allowing efficient and accurate skew removal at any arbitrary clock drop point. The passive transmission medium may be on-chip electrical transmission lines built in current IC technology or possible optical wave-guides in future developments. The proposed technique is naturally insensitive to practical loses and other non ideal effects and has the capability of covering large chip areas.
\end{abstract}

\section{CONVEntional VLSI Clock Distribution}

The common method of distributing $\mathrm{GHz}$ clock signals in VLSI is by active trees. These are hierarchical structures of wires and repeaters, which compensate the limited bandwidths of the wires [1],[2],[3]. Ideally, the signals at the top of the tree are synchronous by physical symmetry but practical errors introduce skews. Table I using data compiled by Rusu [3] shows practical skews of 60 ps or more, independent of technology scaling (quoted papers from different years and technologies) and produced mostly by device mismatches and supply voltage variations [4],[5].

For clocking above $1 \mathrm{GHz}$, regional de-skewing is used to the expense of adding complexity. Specialized circuits mitigate the skew problem [6], [7], but are difficult to migrate to new technology nodes or new products. In some cases, e.g. FPGAs, de-skewing may not be economical.

TABLE I

Performance of skew-corrected active-tree clock distribution schemes (Data compiled by Rusu [3])

\begin{tabular}{|l|c|c|c|}
\hline Author & Source & $\begin{array}{c}\text { Before } \\
\text { De-Skewing }\end{array}$ & $\begin{array}{c}\text { After } \\
\text { De-Skewing }\end{array}$ \\
\hline Geannopoulos & ISSCC-98 & $60 \mathrm{ps}$ & $15 \mathrm{ps}$ \\
\hline Rusu & ISSCC-00 & $110 \mathrm{ps}$ & $28 \mathrm{ps}$ \\
\hline Kurd & ISSCC-01 & $64 \mathrm{ps}$ & $16 \mathrm{ps}$ \\
\hline Stinson & ISSCC-03 & $60 \mathrm{ps}$ & $7 \mathrm{ps}$ \\
\hline
\end{tabular}

Jitter is another important limitation of active trees. It is dominated by the jitter of the repeater, which is proportional to the repeater delay [5]. Since the total repeater delay is about half of the overall tree delay [6], large trees have high jitter.

\section{Clock Distribution Using Transmission Lines}

Recent research results have demonstrated clock distribution networks based on transmission lines (TL) [8],[9],[10]. Here the "wires" are larger in size, operating in "LC-mode" with low loss. The clock signals propagate over much larger distances compared to the conventional tree, without the need of repeaters. The total delay, jitter, and power dissipation reduce considerably. For example, a tapered twolevel H-tree was demonstrated at $5 \mathrm{GHz}$ with 20 ps uncompensated overall skew [8]. This experiment had only 16 clocking zones over a $10 \mathrm{~mm} \times 10 \mathrm{~mm}$ chip area, with a TL characteristic impedance at the top of the tree of only about $4 \Omega$. Expanding this $\mathrm{H}$-tree to 4-5 levels needed in practice would be rather difficult..

A superior use of TLs was reported in [9] and [10] where the circuits achieve about 1 ps skews at $10 \mathrm{GHz}$, with very low power dissipation, while being capable theoretically to cover practical-size clocking regions. Both schemes use many coupled oscillators interconnected into two-dimensional grids. Traveling-wave and standing-wave configurations are used respectively. One limitation of these approaches is having a "hard-wired" operating frequency, determined by the TL characteristics and the capacitive loading. Furthermore, the behavior of such complex autonomous clocking networks in a noisy chip environment is largely unknown. This includes the possibility of exciting multi oscillatory modes creating a potential reliability problems.

The traveling/standing wave concepts have additional limitations. Under traveling-wave conditions, the signals derived at different locations along the TL have a constant magnitude but different phases, which vary linearly with position. De-skewing the clock drop-points is mandatory but fundamentally difficult without an absolute phase reference. Under standing-wave conditions, signals extracted at different locations along the TL have the same phase but different magnitudes. These vary as a sinusoidal function with position creating sizable regions where the signal is too small for practical clock extraction.

Our new concept for TL-based clock distribution has equal capabilities for jitter and power dissipation performance as in [9] and [10] by similarity of construction, but unlike these techniques, it is an open-loop method (no oscillators) generating constant-phase and constant-magnitude clock signals simultaneously. In addition, it is a wide band design not requiring resonating inductors as in [11]. Next, we introduce our technique systematically, first with a review of single-line passive serial distribution, then describing the concepts of bidirectional signaling and average time extraction, and finally, developing our simplest and most promising clocking scheme using analog multipliers.

\section{PAssive Serial Distribution}

The classical active H-tree to be replaced is shown in Fig. 1(a). Our scheme uses passive serial connectivity as illustrated in Fig. 1(b). An on-chip transmission line properly terminated at both ends, meanders over the area to be clocked with buffers tapping the line and deriving local clock signals. Ignoring for the moment the major issue of variable skew 


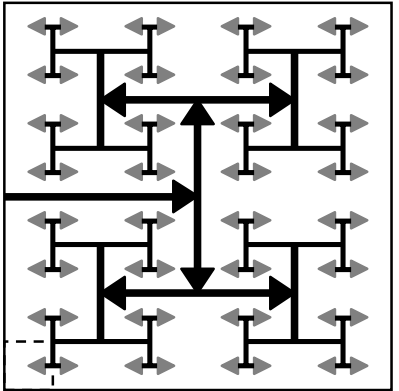

a)

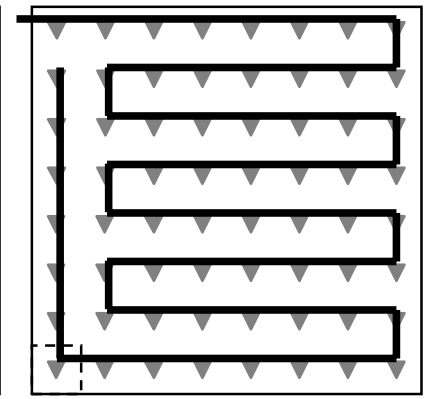

b)
Fig. 1. Clock distribution networks: a) active "H" tree; b) passive serial

along the line as in [9], we notice that there are fundamental reasons why this connectivity is ideally suited for clock distribution.

First, the serial connectivity allows full topological flexibility. There are many ways to connect the local clocking regions without any need for symmetry or regular patterns as in a tree. Second, as discussed before, the passive nature of TLs eliminates the need for repeaters, with minimum power dissipation and jitter. Third, not having branches as in [8] maintains a constant characteristic impedance.

Next, we discuss the loading effects. The input impedance of the buffers tapping the line is purely capacitive in practice. The finite resistance "seen" into the transistor gates at the clock frequency $f_{\text {clock }}$ is negligible because the clock frequency is always low compared to the MOSFET transient frequency $f_{T}$. For example, $10 \mathrm{FO} 4$ clocking gives a $f_{T} / f_{\text {clock }}$ ratio of about 40. Therefore, the buffer loading will not introduce any significant signal loss. However, it is important to avoid reflections, which could introduce magnitude variations.

If the buffers have small input transistors the loading effects are negligible. This would be a first design choice. For larger buffer input transistors, the effective characteristic impedance of the line changes locally, but if all buffers are equal in size, there is only a global effective characteristic impedance change. This can be easily compensated by adjusting the line terminations accordingly by design. A more sophisticated solution would set the termination impedance adaptively by monitoring the signal levels at the TL ends, intentionally placed in close proximity of each other.

The remaining major issue is the severe problem of variable skews on serial distribution. These skews are predictable due to constant speed of EM waves ("light") in the TL. However this does not help much with de-skewing locally extracted clocks. In the absence of a locally available absolute reference, which is the very purpose of clock distribution in the first place, the only theoretical option is to synchronize adjacent areas in a hierarchical manner with a large number of PLLs or DLLs. The resulting complexity and performance limitations would likely be worse than for active trees. We will show that there is another much simpler solution to this synchronization problem if the single TL is replaced by two TLs.

\section{Bidirectional Signaling AND Average Time EXTRACTION}

Consider two identical TLs side-by-side, as shown in Fig. 2 , with two respective pulses originating from opposite ends at the same time. If the bi-directional pulses traveling through the TLs are sensed at any coordinate $x$, two respective skews $T 1$ and $T 2$ are detected. The simple operations of adding or averaging these skew numbers yield absolute results independent of $x$. For example, the $1 / 2(\mathrm{~T} 1+\mathrm{T} 2)$ average equals the time of flight on any of the two signals to the center of the line, which is a constant as illustrated in Fig. 2. Likewise, the $(\mathrm{T} 1+\mathrm{T} 2)$ sum represents the total propagation time over the length L. If multiple points in the line extract and process the bi-directional skews as mentioned, all local clock signals would be phase synchronous. This concept, to be called BDS for Bi-Directional Signaling, enables serial passive clocking theoretically. A circuit extracting the absolute reference from the BDS line will be called an Average Time Extractor or ATE.

It is important to notice that the BDS principle holds even if one adds an arbitrary fixed delay between the starting times of the two signals. Since there is no need to keep the two sources in a predetermined phase relationship, one of them, i.e., the one at the "far end" could be replaced by a PLL or DLL regenerating the signal coming from the first line. This decouples the two ends allowing complete freedom in line placement. Furthermore, the "far-end" source may be eliminated altogether and the signal just wrapped around, as shown in Fig. 3a.

The realization of the ATE function would run into fundamental difficulties (non-causal system) if we had only a single pair of pulses. Fortunately, the distributed clock is a periodic signal and therefore the ATE can average "future" values from the previous clock period. Many ATE configurations are possible. The block diagram in Fig. 3b shows a topology based on a classical DLL. The feedback controls the delay elements forcing the total delay to be equal to the time interval between the arrival times of the two input pulses. In lock state, the center tap of the delay line extracts the absolute time reference $1 / 2 \mathrm{~T} 0$. The delay control could be

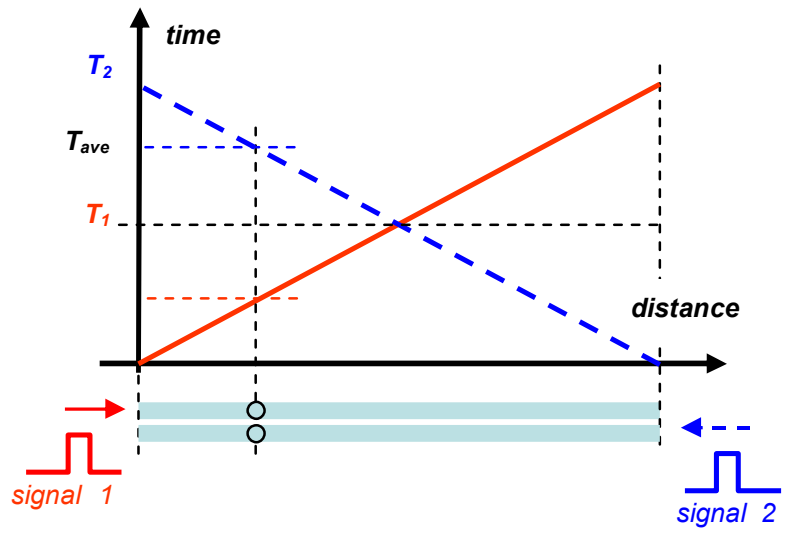

Fig. 2. Word-line (time vs. space) diagram of a BDS system. 


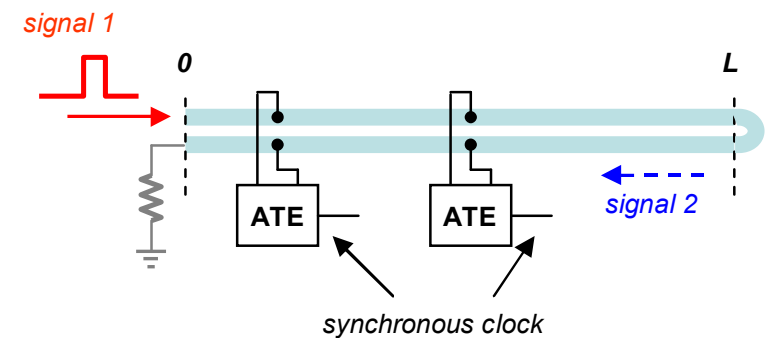

a)

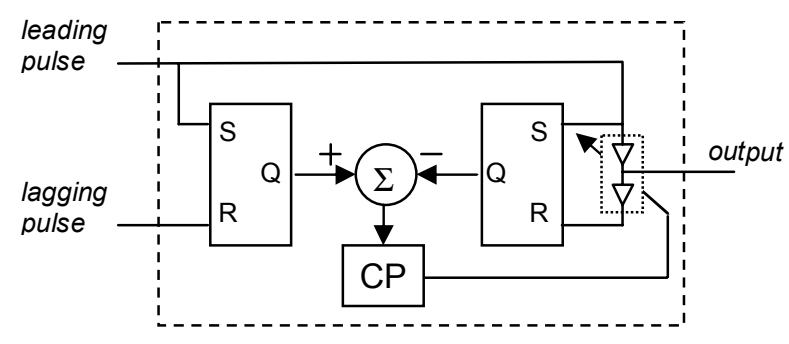

b)

Fig. 3. Conceptual schematic of pulse-reference BDS system: a) folded-line architecture; b) possible ATE implementation;

either analog or digital. For the latter, the DLL could be disabled after lock. A transistor-level circuit for this ATE and a similar PLL-based topology were designed in $0.13 \mathrm{u}$ TSMC CMOS and the correct operation was verified in simulations at multi-GHz operation. We leave any quantitative claims after test chips are fabricated and tested.

The ATE function as defined for periodic signals cannot distinguish between delays separated by an integer number of the clock period. This gives rise to a phase reversal ambiguity problem. In other words, while all ATEs connected to the BDS line are synchronized in terms of clock transitions, some ATEs will have inverted signals with respect to others, depending on the ATE position along the TL. This consistent phase inversion error, occurring over large TL sections, can be easily detected during design and corrected with the addition of inverters. If it is desirable to allow blind ATE placement with no attention to potential phase reversals, it is possible to design simple circuits, which would correct this error automatically. For example, at the boundary of each clocking region, a phase detector would check if the two regional clocks are in phase or out of phase and communicate this to an appropriate local clock driver through a single control bit. This one time operation at turn on is considerably easier than complicated boundary phase comparison and correction for clock-transition edge alignment already used in VLSI with high precision DLLs.

A more problematic practical issue is the phase error introduced by pulse shape distortion due to dispersion or any other mechanism. This is an important potential problem for any ATE processing high frequency pulses. As discussed previously, the clocking speed is low compared to the transistor switching speed but still the demands of modern clocking systems are very stringent. Next, we show a superior solution to the clock extraction problem from a BDS line, which is considerably simpler than the ATEs discussed so far, has no phase ambiguity, and does not rely on edge detection. This is accomplished by using sinusoidal BDS and multipliers for clock extraction.

\section{SINUSOIDAL BDS SYSTEM AND ClOCK EXTRACTION BY MULTIPLICATION}

For sinusoidal signals, delay and phase summation are equivalent operations. Indeed, multiplying two sinusoidal signals of frequency $f$ and phases $\varphi 1$ and $\varphi 2$ yields a DC term and a sinusoidal term of frequency $2 f$ and phase $(\varphi 1+\varphi 2)$. But, $\varphi 1$ and $\varphi 2$ are proportional to corresponding time delays. Therefore, the ATE functionality is automatically included in the operation of analog signal multiplication.

We can configure a very efficient BDS clock distribution system as in Fig. 4a.. A single sinusoidal signal of frequency $f$ enters the TL at node A, passes sequentially through B, C and $\mathrm{D}$ and exits into a termination resistor at node E. Analog multipliers are connected as shown. After multiplication, phase synchronous local clocks are derived at points $\mathrm{AA}, \mathrm{BB}$, $\mathrm{CC}$, or any additional similar points.

Extracting a clock signal at twice the transmitted frequency is beneficial since the line loss for half-rate distribution is lower (skin-effect limited operation). Half-rate distribution has been used successfully in commercial microprocessors [7], albeit with a conventional active tree.

Unlike using DLLs or PLLs, analog multiplication is a simple memory-less (non-dynamic) operation. This classical, well-understood circuit function can be implemented in any semiconductor technology [12]. The DC term resulting from the multiplication can be easily removed through AC coupling or standard DC removal feedback techniques.

Extensive transient and harmonic-balance simulations of the proposed sinusoidal BDS were performed using the MentorGraphics Eldo-RF simulator. We modeled the BDS line with Level-4 TL elements, assuming an on-chip $\mathrm{Cu}$ microstrip design with $10 \mu \mathrm{m}$ width, $10 \mu \mathrm{m}$ dielectric (SiO2) thickness and $5 \mu \mathrm{m}$ metal thickness. The characteristic impedance was approximately $80 \Omega$. The multipliers were modeled using $120 \mathrm{~nm}$ TSMC CMOS technology files. The total BDS line length in our simulations was $32 \mathrm{~mm}(64 \mathrm{~mm}$ total TL length) with 32 local clocking points, one per every millimeter. Such a BDS line can provide synchronous clocks to a $32 \mathrm{~mm}^{2}$ area of any shape with $1 \mathrm{~mm} \times 1 \mathrm{~mm}$ granularity. Four such lines centrally fed would cover $128 \mathrm{~mm}^{2}$ area.

Fig. $4 \mathrm{~b}$ shows a typical simulation result for $2 \mathrm{GHz}$ distribution and $4 \mathrm{GHz}$ local clocks. For clarity only 3 out of 32 clock signals are shown. We notice the large skews at points B, C, D, and E compared to A, consistent with finite signal propagation speed. After multiplication, all $4 \mathrm{GHz}$ local clocks are in phase synchronism. The different DC levels, a byproduct of the multiplication, are intentionally kept for demonstration purposes. They would be removed in practice, as discussed previously. 


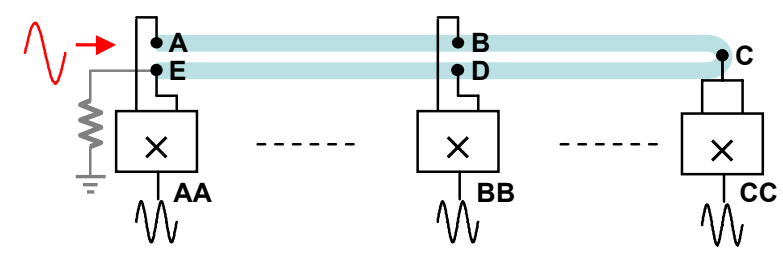

a)

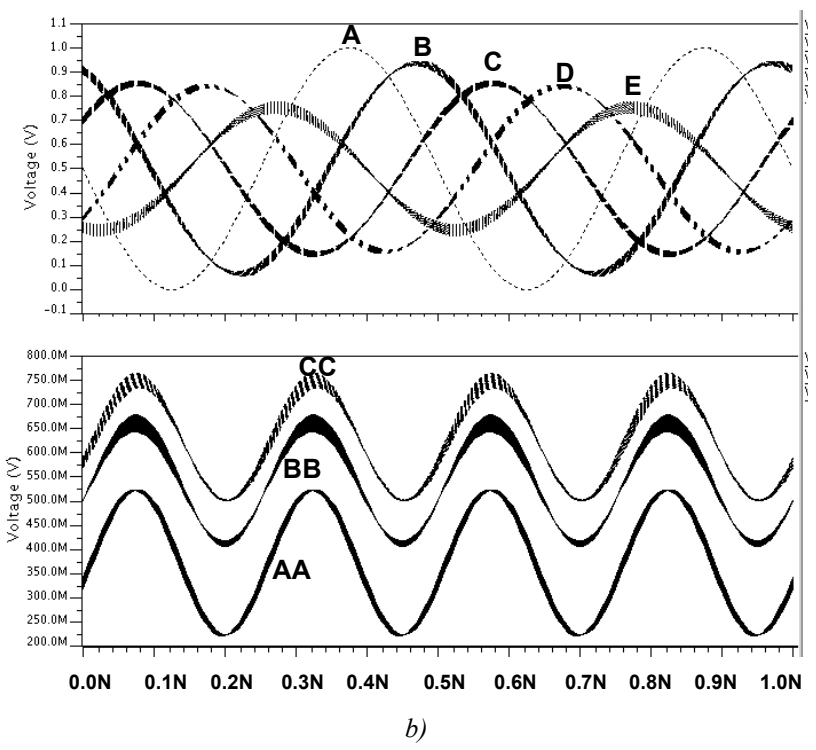

Fig. 4. Sinusoidal BDS system: a) block diagram; b) simulation results;

Several important non-ideal conditions are included in these simulations. First, we have realistic TL loss: approximately $6 \mathrm{~dB}$ from point $\mathrm{A}$ to point $\mathrm{E}$. This loss has little effect on the extracted clocks, confirming a theoretical observation that the extracted clock is independent of TL loss and position. We expect to have no phase errors due to signal loss but on a first guess, we would expect to see magnitude errors. However even the magnitude is insensitive to loss. This important property is a consequence of the fact that the TL loss in $\mathrm{dB}$ is linear with the distance. As multiplication is equivalent to addition in $\mathrm{dB}$, a loss-error cancellation occurs at every clock drop point. The slight inconsequential magnitude error visible in Fig. $4 \mathrm{~b}$ is due to large loading mismatches in the line introduced in simulations creating reflections.

Second, notice that all signal traces are somewhat thick. This is because each one is composed of twenty superimposed traces resulting from a $25 \%$ termination resistance sweep. The synchronization errors are obviously small proving our technique is resilient to termination errors.

Finally, we mention that at every TL-tapping point (there are 64 such points) the multipliers load the line with the equivalent of four minimum CMOS inverters.

\section{CONCLUSION}

We have introduced a general VLSI clock distribution concept using bidirectional signaling on an integrated wave guide. This concept may be implemented with several technology and circuit choices such as using electrical TLs in current IC technologies or optical guides in future IC technologies, and performing clock extraction based on pulse arrival-time averages or analog multiplication. The most attractive practical approach for current IC technology is using electrical transmission lines and analog multipliers. Simulations and theoretical arguments show that this very simple architecture is insensitive to all major practical nonideal effects and has optimum power dissipation and jitter.

\section{ACKNOWLEDGEMENTS}

We thank Applied Materials Inc. and especially Dr. M. Pinto, Dr. L. West and Dr. D. Eaglesham for the support of this work. We also thank Dr. B Ackland of Nobel Device Technologies, our co-author of the BDS concept discovery for many useful discussions.

\section{REFERENCES}

[1] E. Friedman, "Clock Distribution Networks in Synchronous Digital Integrated Circuits" Proc. IEEE, Vol. 89, No 5, pp. 665-692, May 2001.

[2] A. Mule', E. Glytsis, T. Gaylord, J. Meindl, "Electrical and Optical Clock Distribution Networks For Gigascale Microprocessors," IEEE Trans. On VLSI Systems, Vol. 10, No. 5, pp. 582 -592, Oct. 2002.

[3] S. Rusu, "Clock Generation and Distribution for High-Performance Processors" invited presentation, International Symposium on Systemon-Chip, 2004.

[4] G. Geannopoulos, X. Dai, "An Adaptive Digital Deskewing Circuit for Clock Distribution Networks," International Solid-State Circuit Conference Dig. Tech. Papers, pp 400-401, Feb. 1998.

[5] D. Harris and S. Naffziger, "Statistical Clock Skew Modeling with Data Delay Variation," IEEE Trans. VLSI Systems, Vol. 9, No. 6, pp. 888 898, Dec. 2001.

[6] J. Warnock at al, "The circuit and physical design of the POWER4 microprocessor," IBM Journal of R\&D, Vol. 46, Nov. 2002.

[7] P. Mahoney, E. Fetzer, B. Doyle, S. Naffziger, "Clock Distribution on a Dual-Core Multi-Threaded Itanium $\AA$-Family Processor," International Solid-State Circuit Conference, Dig. Technical Papers, pp. 292-293, Feb. 2005.

[8] M. Mizuno at al, "On-chip Multi-GHz Clocking with Transmission Lines", International Solid-State Circuit Conference Dig. Tech. Papers, 2000.

[9] J. Wood, T. Edwards and S. Lipa, "Rotary traveling-wave oscillator arrays: a new clock technology," IEEE Journal of Solid-State Circuits, Vol. 16, pp. 1654 - 1665, Nov. 2001.

[10] F.O'Mahony, P. Yue, M. Horowitz and S. Wong, "A 10-GHz Global Clock Distribution Using Coupled Standing-Wave Oscillators," IEEE Journal of Solid-State Circuits, Vol. 38, No. 11, pp. 1813-1820, Nov. 2003

[11] S. Chan, K. Shepard, P. Restle, "Uniform-Phase Uniform-Amplitude Resonant Load Global Clock Distributions" IEEE Journal of Solid-State Circuits, Vol. 40, pp. 102 - 109, Jan. 2005.

[12] G. Han and E. Sanchez-Sinencio, "CMOS Transconductance Multipliers: A Tutorial," IEEE Trans. Circuits and Systems - II, Vol. 45, No. 12, pp. 1550 - 1563, Dec. 1998. 